\title{
Low-cost interrogation system for dynamic sensor applications using a resin based fiber Fabry Perot filter
}

\author{
João Preizal, Lúcia Bilro, and Ricardo Oliveira* \\ Instituto de Telecomunicações and Universidade de Aveiro, Campus Universitário de Santiago, 3810-193 Aveiro, Portugal
}

\begin{abstract}
In this paper it will be presented a prototype of a low-cost power interrogator for dynamic sensor applications. The proposed system is based on the edge filter technique, where the slope of an in-house resin based Fabry Perot (FP) filter is used to actively filter the power of a narrowband laser centred at the middle part of the FP slope region. The interrogator is composed of a laser source, circulator, photodetector, Arduino board and laptop. Tests have been performed in order to check the viability of the system. Finally, the measurement of a pulse wave signal has been performed by embedding the FP sensor in a 3D printed elastic membrane and reading the signal through the proposed interrogator.
\end{abstract}

\section{Introduction}

Fiber optic sensors are a technology that has been developed in many different areas in the last century. They present several qualities, such as durability in the most diverse environments, immunity to electromagnetic fields, compatibility, lightweight, ability to operate in long distances, they are compact, could be implemented with multiplexing capabilities, etc.

Most of the sensors reported in literature, such as fiber Bragg gratings and a variety of interferometers, rely on the wavelength detection technique. However, for such measurement schemes, it is required the use of bulk, and expensive fiber interrogators [1]. Furthermore, the acquisition frequency is normally low which is problematic when dynamic applications are required. Thus, here we propose the creation of an interrogator based on the edge filter technique [2], capable to read the data through a photodetector in an easy, simple and cost effective way. In order to keep the cost low, fiber Fabry Perot interferometers (FPIs), based on the resin splice method [3] will be used.

In this work we propose a simple and low-cost intensity detection system operating with an in-house FPI cavity. The edge filter technique will be used and the power fluctuations generated by the filter will be measured through a photodetector that is attached to the analogical input of an Arduino board. The dynamic response of the sensor will be tested for different frequencies and to finalise the system will be used to measure the pulse wave signal at the wrist region.

\section{Theoretical concept}

The theoretical basis of this paper is mainly based on the study of the power variation for a given wavelength, using the edge filtering technique. In this technique, a narrowband laser is actively filtered by an external filter.

The edge filter will filter the optical signal coming from the laser source and will limit the output power according to the spectral response of the filter at the laser wavelength location, as it can be seen on Fig. 1Erro! A origem da referência não foi encontrada..

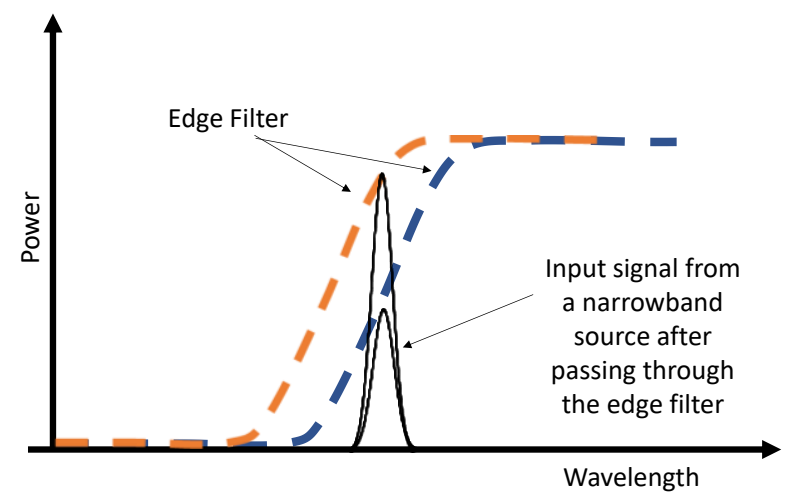

Fig. 1. Power fluctuation of a laser source as it passes through an edge filter.

In order to keep the cost of the system low, while keeping its simplicity, we decide to implement an FPI filter fabricated through the resin based method [3]. The spectral response of this filter varies between minima and maxima along the wavelength, being the transitions between minima and maxima well adapted to act as an edge filter. The wavelength range of this region is however controlled by the cavity length developed and thus, can be designed according to the desired need. When this FPI is implemented through the edge filtering technique, it is able to actively filter the incoming signal by simply adjusting the location of the filter, that is possible to be performed by adjusting the cavity length, e.g., strain, curvature, temperature, etc. The operation

\footnotetext{
$\overline{\text { *Corresponding author: oliveiraricas@av.it.pt }}$
} 
principle of the edge filtering technique implemented with a FPI may be seen in Fig. 2.

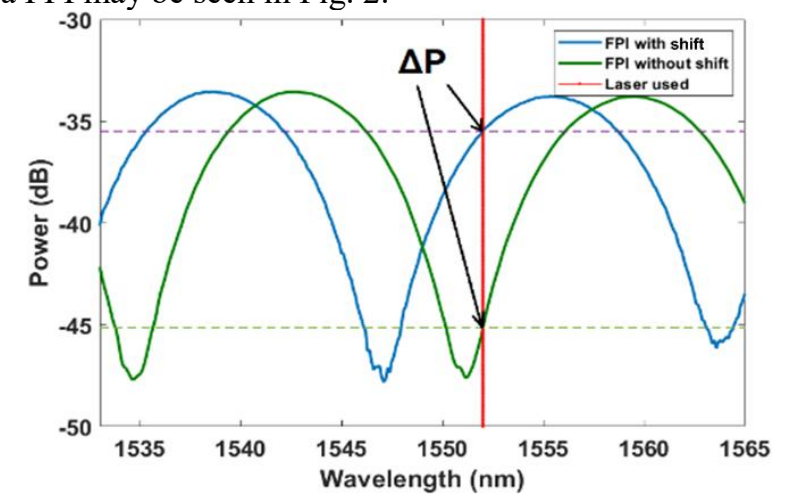

Fig. 2. Interference spectrum of the FPI cavity, showing the region where the laser operates and the correspondent power that can be filtered by the edge of the FPI, when the FPI is under strain.

As it is possibly to verify on Fig. 2, the laser power changes $\Delta \mathrm{P}$, when the FPI spectrum is shifted in wavelength (e.g., when subjected to an external load).

\section{Methods and experimental setup}

Firstly, we experimentally validate the proposed technology by measuring the movement of the membrane of a speaker that has been subjected to different sinusoidal waves. For that, it was used a resin based FPI sensor fabricated through the technique described in [3], [4], where two single mode fibers aligned transversely to each other and with a small longitudinal distance in between, are cold-spliced with a ultraviolet curable resin. To allow the FPI to read the oscillatory movement of the membrane on the speaker, one of the terminals of the optical fiber is glued perpendicular to it, while the other is used as the In/Out terminal, as is shown in Fig. 3Erro! A origem da referência não foi encontrada.. With this configuration, the cavity length could change as the membrane of the speaker goes up and down according the wave generated on the speaker. This will induce a shift in the spectrum of the FPI cavity that actively filters the power of the laser.

The signal detection is made in reflection using an optical circulator. For that, a narrowband laser centred at the linear region of the FPI travels up to the. The reflected power (already filtered) is fed into a photodetector through the output port of the circulator. The optical signal is converted to the electrical domain by the photodetector, and an Arduino board is used to read and to perform the digitalization of the signal. The data is transferred to a computer and finally, it is represented in real time through a dedicated graphical user interface. An FFT is also applied to the data allowing to get the frequency of the oscillation. The full schematic of the operation principle of the proposed interrogator and detection scheme may be seen in Fig. 3.

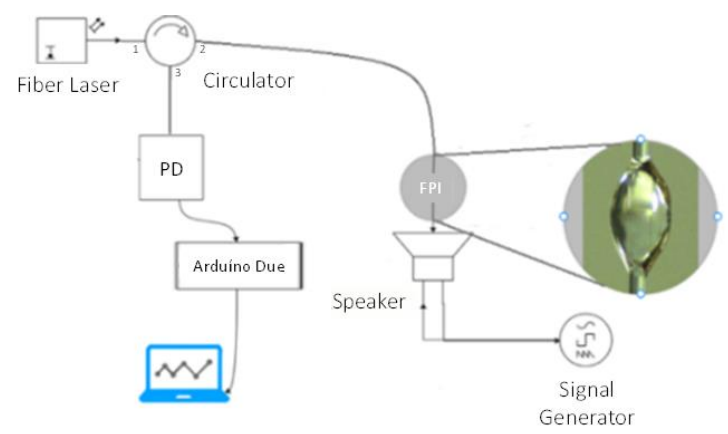

Fig. 3. Schematic of the operation principle. It is composed of a detection system on the left and by an FPI sensor attached to the membrane of a speaker on the right. The inset shows the microscope image of the resin based FPI sensor.

The data acquisition boards that compose this interrogator (i.e. circuit for the photodetector board and for the Arduino board) are assembled in a 3D printed box, allowing to get a more compact system for easy access. The final result of the interrogation system may be seen in Fig. 4Erro! A origem da referência não foi encontrada.
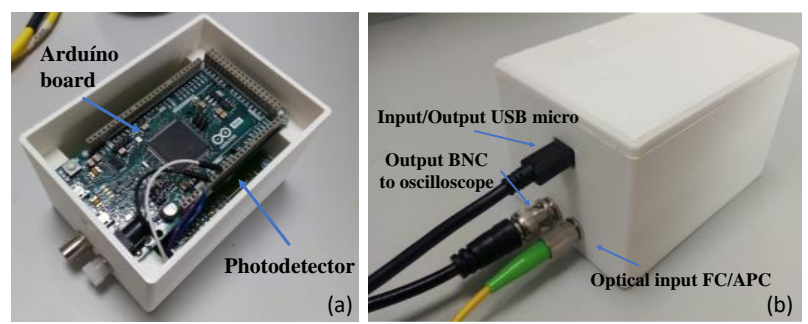

Fig. 4. (a) Inside and (b) outside picture of the data acquisition boards that compose the interrogator.

In Fig. 4, it is possible to observe that the front panel of the interrogator box contains three different connection ports, namely one FC/APC optical adaptor for the reading of the optical signal, one micro USB port to export data to a computer, and finally, a BNC port for ready visualization of the signal using an oscilloscope.

In a second stage of the work, we decided to implement the same detection scheme for the measurement of a pulse wave at the wrist region. For that, the FPI sensor was embedded on an elastic membrane that is latter attached to a fabric bracelet, allowing it to be stretched and adjusted to the wrist of different individuals. The computer aided design image of the elastic membrane may be seen in Fig. 5(a), while the fully developed sensor on Fig. 5(b).
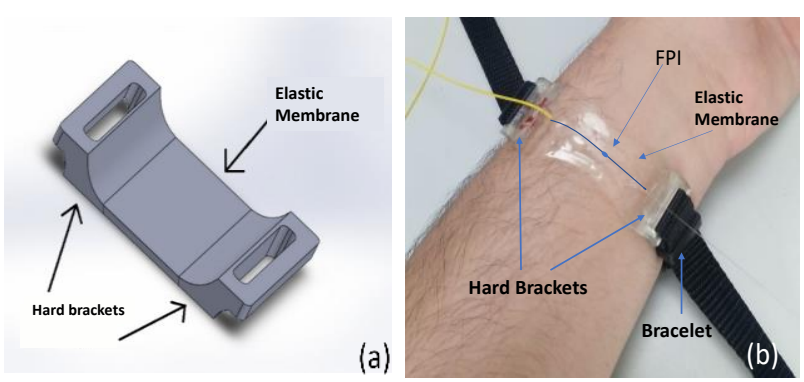

Fig. 5. (a) Drawing of the part used to measure the pulse wave and, (b) how it was implemented on the wrist region. 
The principle behind the detection of the pulse wave relies on the blood pressure signal generated at the wrist region. Thus, the FPI embedded on the elastic membrane is subjected to a longitudinal strain, allowing it to change the cavity length and shift the spectrum according to the pulse wave, generating a power fluctuation during time.

\section{Results and discussion}

For the first part of the work, the data was taken for frequencies below and above $1 \mathrm{~Hz}$. An example of the results taken for the $0.5 \mathrm{~Hz}$ and $2.4 \mathrm{kHz}$ may be seen in Fig. 6(a) and (b), respectively.
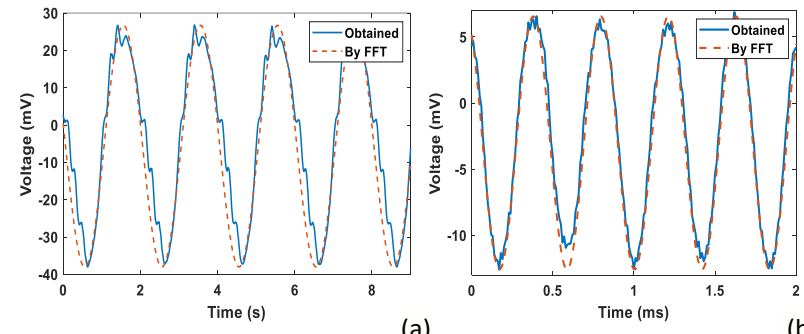

(a)
Fig. 6. Data taken for (a) $0.5 \mathrm{~Hz}$ and (b) $2.4 \mathrm{kHz}$

As shown in Fig. 6(a) and (b), the raw signals (blue traces), show a clear sinusoidal behaviour, that is in agreement with the movement performed by the membrane of the speaker. However, the signal also shows some noise, that is associated to the environmental conditions, such as noise from the equipment's. By performing an FFT to the data shown in Fig. 6, it is possible to calculate the frequency of the signal. Using that, we were able to reconstruct the signal as is shown by the red dotted lines.

The frequency collected from the FFT was also used to check the response of the sensor for a span of frequencies and the results may be seen in Fig. 7. From that, it is possible to observe that the sensor has a flat response up to $500 \mathrm{~Hz}$. Then, the amplitude decreases possibly due to the elasticity of the membrane itself. However, the bandwidth fits quite well a variety of applications.

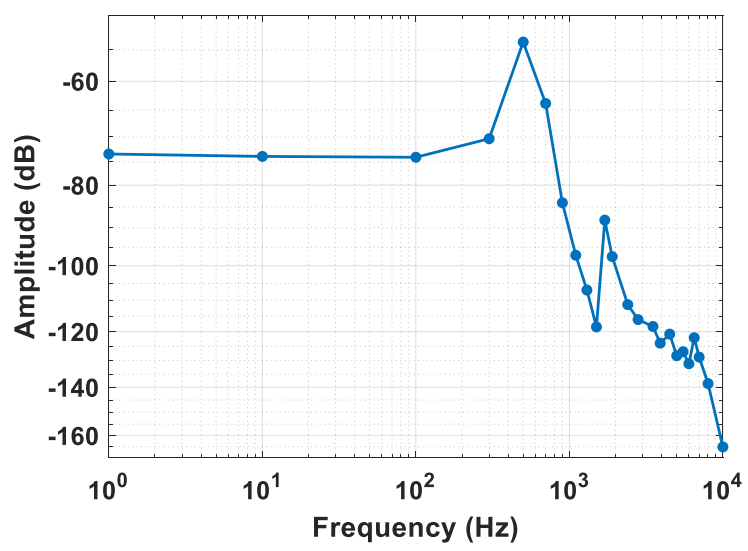

Fig. 7. Frequency response of the proposed fiber sensor.

The prototype was then experimentally tested as a wearable device for the monitoring of radial pulse wave.
Despite the difficulties in acquiring the data, possibly due to the elasticity of the membrane, the results were similar to the expected ones, where an initial peak is achieved (main wave), followed by a dip (dicrotic notch), as is shown in Fig. 8.

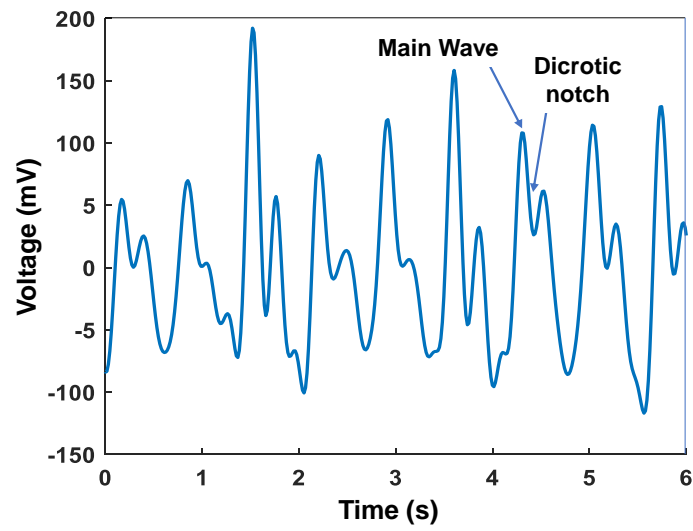

Fig. 8. Measured pulse wave signal at the wrist region.

The results revealed that the proposed fiber sensor can be used to measure the pulse wave signal in a simple and cost effective way.

In the overall we have shown that it is possible to transduce measurements commonly performed in the spectral domain, to simple power detection schemes that could be used with cost effective devices, paving the way for a simpler and cost effective use of fiber optic sensors.

\section{Conclusions}

In this work a low-cost fiber optic interrogation system has been implemented. The system uses an FPI sensor to actively filter the output power of a narrowband laser. By subjecting the FPI sensor to external conditions, such as load, the interference spectrum is wavelength shifted producing a power change that is then detected through a photodetector attached to an arduino board. The results showed that the proposed system has a flat response up to $500 \mathrm{~Hz}$ and that it could be used for instance in the detection of the pulse wave at the wrist region.

This work was funded by FCT-Fundação para a Ciência e Tecnologia and by the European Regional Development Fund (FEDER), through the Competitiveness and Internationalization Operational Programme (COMPETE 2020) of the Portugal 2020 framework and by national funds under the projects FOPEComSens (PTDC/EEI-TEL/1511/20), AQUATICsens (POCI-01-0145-FEDER-032057) and UIDB/50008/2020UIDP/50008/2020.

\section{REFERENCES}

[1] A. Cusano, A. Cutolo, and J. Albert, Fiber Bragg Grating Sensors: Research Advancements, Industrial Applications and Market Exploitation, 1st ed. Bentham Science Publishers Ltd., 2011.

Y. J. Rao, Z. L. Ran, and Y. Gong, Fiber-optic fabry-perot sensors: An introduction. 2017.

[3] R. Oliveira, L. Bilro, and R. Nogueira, "Fabry- 
Pérot cavities based on photopolymerizable resins for sensing applications," Opt. Mater. Express, vol. 8, no. 8, pp. 899-902, 2018.

[4] R. Oliveira, L. Bilro, T. H. R. Marques, C. M. B. Cordeiro, and R. Nogueira, "Simultaneous detection of humidity and temperature through an adhesive based Fabry - Pérot cavity combined with polymer fiber Bragg grating," Opt. Lasers Eng., vol. 114, pp. 37-43, 2019. 